\title{
Y SI NOS OLVIDAMOS \\ DE LA DETECCIÓN DEL TALENTO... \\ Y SI INDIVIDUALIZAMOS EL PROCESO \\ DE DESARROLLO DE SU TALENTO
}

\section{And if we forget the talent detection... And if we individualize the development of HIS Talent}

\author{
Alberto Lorenzo*, Jorge Lorenzo* y Sergio JiMÉneZ** \\ * Facultad de Ciencias de la Actividad Física y del Deporte - INEF. \\ Universidad Politécnica de Madrid \\ ** Facultad de Ciencias de la Actividad Física y el Deporte. \\ Universidad Europea de Madrid \\ Correo-e: Alberto.lorenzo@upm.es
}

Recepción: 9 de noviembre de 2014

Envío a informantes: Io de noviembre de 20I4

Aceptación definitiva: 17 de febrero de 2015

Biblid. [0214-3402 (2015) (II época) n. ${ }^{2}$ 21; 105-I27]

Resumen: Durante muchos años se ha entendido el proceso de detección de talentos en el ámbito deportivo como un proceso cercano a lo mágico (aún se entiende en muchas ocasiones así), según el cual, el entrenador era capaz de, mediante ciertas baterías de test, poder predecir el futuro de los deportistas, y discernir entre todas ellas quién era la persona agraciada que iba a ser campeona del mundo. ¿Qué ideas y argumentos pueden llevar a pensar que esto es posible? Del mismo modo, bajo la misma idea del desarrollo del talento, también se pensaba que, conociendo cómo son los deportistas que han alcanzado el máximo rendimiento posible, también sería posible diseñar un proceso de entrenamiento perfecto que permitiese a los más jóvenes, si cumplían perfectamente con el camino establecido, alcanzar o incluso superar dicho rendimiento. Pero, ¿̇realmente pensamos que esto es posible?

Lejos de aceptar dichas ideas (y las que la sustentan principalmente), hemos de considerar que son muchas las personas que presentan el potencial para poder obtener rendimientos elevados en el ámbito deportivo. Y que lejos de pensar que tan solo son unos los agraciados por gracia del destino o la lotería genética, debemos entender que, si somos capaces de controlar y favorecer el proceso de desarrollo de una persona, estaremos más cerca de conseguir que se alcancen los niveles de rendimiento deseados. ¿Qué es lo que permite o provoca que un deportista sí alcance 
los resultados esperados y otros no? ¿Qué factores favorecen el desarrollo del deportista? ¿Cómo diseñar el proceso de desarrollo del deportista? Pretendemos en el presente artículo desgranar las principales ideas y argumentos con los que se afronta el proceso de detección y desarrollo del talento, así como ofrecer al lector algunas ideas que le ayuden a mejorar el proceso formativo de cualquier deportista que pueda caer en sus manos.

Palabras Clave: detección del talento; desarrollo del talento; transfer talent; talent recycling; mature-age talent identification.

ABSTRACT: For many years, it has been understood that the process of sport talent identification as a close to magic process (although it is understood in many cases as well), in which the coach was able, by some tests and test batteries, able to predict the future of athletes, and discern among them, who are the graceful people that would be World Champions. What ideas and arguments can lead to think that this is possible? Similarly, under the idea of talent development, it is also thought that, knowing how these athletes who have achieved the highest possible performance, it would also be possible to design a perfect process that allowed younger, if they met perfectly with the path set, reach or even exceed that performance. Do we really think this is possible?

Far from accepting such ideas (and that support mainly), we must consider that there are many people who have the potential to produce high yields, in our case in sports. And far from thinking that you are just a graceful by grace of fate or the genetic lottery, we must understand that if we are able to monitor and facilitate the development process of a person, will be closer to being able to achieve the desired levels of performance. What is it that allows or causes an athlete to achieve the expected results when others do not? What factors favor the development of the athlete? How to design the development process of the athlete? We aim in this article summarize the main ideas and arguments with which the detection process and developing talent is addressed, and give the reader some ideas to help improve the learning process of any athlete who may fall into their hands.

KEY WORDS: talent tetection; talent development; transfer talent; talent recycling; mature-age talent identification.

\section{Introducción}

$\longrightarrow$ UANDO UNO SE ENFRENTA Al MUNDO DE LA DETECCión del talento o del desarrollo del talento, no es capaz de imaginar la cantidad de conceptos relacionados entre sí existentes, ni la multitud de perspectivas con las que se puede afrontar su problemática. Dicha situación supone una gran complejidad y dificultad para entender el proceso correctamente, al mismo tiempo que genera, en muchas ocasiones, ideas o conceptos que no se corresponden con la realidad. Y esto es lo primero que debemos tener claro. Nos encontramos ante un proceso muy complejo y, por más que intentemos explicarlo a partir de teorías, ideas, clasificaciones y factores con la idea de simplificar y reducir la complejidad de las cosas, no debemos perder de vista el hecho de que el mundo es mucho más aleatorio de lo que nos gustaría.

En esta problemática, además, siempre subyace el eterno debate entre la perspectiva genetista y la visión ambientalista, entre el paradigma cuantitativo y cualitativo. 
Desde la perspectiva genetista, se asocia la palabra talento a esa capacidad transmitida genéticamente e innata con la que el deportista es bendecido, y que, a partir de ciertos indicadores previos, permite a gente experta identificar la presencia del talento antes de alcanzar elevados rendimientos. Estas primeras indicaciones del talento constituyen una base para predecir la posibilidad de obtener rendimiento (Howe, Davidson y Slovoda, 1998).

Desde la perspectiva ambientalista, sin embargo, se considera a la persona como una pizarra en blanco a la que cualquier cosa que ocurra después de su nacimiento es consecuencia de su experiencia y aprendizaje. Bajo esta perspectiva, se han identificado varios factores que contribuyen al desarrollo de los deportistas expertos (e. g., Lorenzo y Calleja, 20Io; Lorenzo y Sampaio, 2005; Gulbin, Oldenziel, Weissensteiner y Gagné, 20ıо; Li, John Wang y Young Pyun, 20ı4; Sáenz-López, Ibáñez, Giménez, Sierra y Sánchez, 2005). Simonton (I999) señala que es muy probable que los factores ambientales, incluida la práctica deliberada, provoquen más variación en el rendimiento que las capacidades innatas del sujeto talentoso en cualquier dominio. Estos estudios fundamentalmente se han desarrollado bajo el paradigma experto-novato y bajo el auspicio de la metodología cualitativa.

Sin embargo, para avanzar en el conocimiento del desarrollo de la pericia y del talento, es preciso adoptar una perspectiva multidisciplinar e integradora, donde las diferentes perspectivas y ciencias aporten sus metodologías y resultados (Phillips, Keith, Renshaw y Portus, 20Io). Lejos de entender el proceso de detección de talentos como la posibilidad de identificar a un futuro deportista a partir de la aplicación de test y lejos de entenderlo como el desarrollo de una herramienta perfecta para valorar el rendimiento de las personas, es preciso valorar más el camino individual seguido por cada deportista y valorar más los momentos precisos para desarrollar el proceso de entrenamiento adecuado a cada edad.

Por ello, antes de avanzar, tenemos que entender que el talento es algo más que una característica muy por encima de la media. El talento aparece por la combinación de diversos componentes innatos (Abbott y Collins, 2004; Simonton, 1999). Es decir, el talento no es un concepto unidimensional, sino que debe ser entendido y explicado a partir de modelos multifactoriales, es un concepto multidimensional y multiplicativo (Burguess y Naugthon, 20Io; Simonton, 200I), y nunca sumativo (es decir, no es el resultado de la suma de todos los factores, sino que es multiplicativo, en el sentido de que la ausencia de ciertas características fundamentales hace que el talento sea nulo). También debe ser entendido como un concepto dinámico, que evoluciona a lo largo del tiempo, a medida que se produce la interacción entre las características del deportista y su contexto (e. g., Gilar y Castejón, 2003; Simonton, I999, 200I), y que exige entender que las características del deportista van evolucionando a lo largo del tiempo.

Finalmente, hay que asumir también que es un constructo social y no natural. Esto se debe, en primer lugar, a que son otros los que deben reconocer que alguien tiene talento. Además, culturalmente debe ser reconocido, debe existir una estructura sobre el campo en cuestión.

\section{Realmente, ¿podemos predecir el futuro?}

¿Cómo se ha abordado tradicionalmente la detección del talento? Este tipo de análisis se ha realizado a partir de dos diseños de estudio diferentes. Por un lado, se ha 
tratado de determinar el perfil idóneo del deportista a partir de la observación de las necesidades del deporte y de las características de los deportistas expertos, basándose en el análisis de características fundamentalmente genéticamente dependientes (e. g., Bourgois et al., 200o; Reilly, Bangsbo y Franks, 2000a; Stroyer, Hansen y Hansen, 2004).

Por otro lado, teniendo en cuenta las características del deporte, se han diseñado baterías de tests para valorar diferentes características o factores que condicionan de forma relevante el rendimiento y que sirviesen para discriminar al deportista con talento y, de esta forma, encontrar a futuros sujetos que destacasen en la especialidad deportiva (e. g., Hoare y Warr, 20oo; Elferink-Gemser, Visscher, Lemmink y Mulder, 2007; Falk, Lidor, Lander y Lang, 2004; Gabbet, Jenkins y Abernethy, 20II; Lidor et al., 2005a; Lidor, Melnik, Bilkevitz, Arnon y Falk, 2005b; Lidor, Hershko, Bilkevitz, Arnon y Falk, 2007; Matthyss et al., 20II; Morris, 2000; Reilly, Bangsbo y Franks, 2000a; Reilly, Williams, Nevill y Franks, 20oob; Torres-Unda et al., 20I3; Vaeyens et al., 2006).

A pesar de los numerosos intentos realizados en este ámbito, esta línea de investigación tiene importantes limitaciones que hacen preciso tomar con cautela los distintos resultados obtenidos. En primer lugar, el propio diseño de las investigaciones provoca que los resultados obtenidos deban ser interpretados con mucha cautela, bien por el tipo de poblaciones utilizadas en el diseño (normalmente realizadas sobre el paradigma experto-novato o con distinto nivel de rendimiento); bien por el tipo de diseño (son muy escasos los estudios longitudinales, utilizándose normalmente diseños transversales o cuasilongitudinales y utilizando para ello poco tiempo en la toma de datos) (Lidor, Côté y Hackfort, 2009).

También es preciso observar que la detección del talento, tal y como se ha entendido durante mucho tiempo, implica necesariamente una predicción. Este postulado es erróneo, ya que sólo es posible predecir un nivel de rendimiento con un margen de error aceptable, si el pronóstico se basa en una marca alcanzada cuando el deportista está cerca de la edad de su madurez. La eficacia en la predicción de este tipo de modelos de detección es inversamente proporcional al tiempo y a la edad sobre la que se intenta realizar la predicción. Los datos ofrecidos por los diferentes estudios confirman que no hay correlación entre los resultados obtenidos en los tests y la selección final de los deportistas (e. g., Lidor et al., 2005a, 2005b; Lidor, Côté y Hackfort, 2009; Vandorpe et al., 20I2).

Entre los factores que tienen una influencia decisiva sobre el valor final del rendimiento se encuentran el entrenamiento y el grado de maduración. En relación al entrenamiento, Pearson, Naugthon y Torode (2006) muestran de forma particularmente clara que la configuración de las aptitudes exigidas para triunfar en una tarea se transforma en el curso del aprendizaje y con la influencia del entrenamiento. Esto hace que sea cuestionable que la identificación del talento se pueda hacer a partir de dichas variables, ya que evolucionan a lo largo del tiempo y parece algo irreal el valorar el rendimiento futuro a partir de dichas variables (Pearson et al., 2006; Williams y Reilly, 200o).

Por otro lado, la mayoría de las cualidades que distinguen a los deportistas de elite en la edad adulta no aparecen hasta los últimos estadios de la adolescencia (Vaeyens, Lenoir, Williams y Philippaerts, 2008). Al variar las características y aptitudes de los deportistas a lo largo del tiempo, los valores que discriminan a los sujetos varían en 
función de la edad y del grado de maduración y, por tanto, existen diferentes indicadores de rendimiento que caracterizan el éxito en diferentes edades (por ejemplo, las variables cineantropométricas van disminuyendo su importancia e influencia en el rendimiento a lo largo de los años). Una clara consecuencia de esto es que es preciso diseñar baterías de test asociadas a cada edad, que valoren distintos indicadores de rendimiento en función de la edad biológica (e. g., Pienaar y Spamer, 1998; Vaeyens et al., 2006).

Esta influencia de la maduración ha de ser tenida también en cuenta en el diseño de la investigación, de tal manera, que en vez de comparar a deportistas con la misma edad cronológica, deberían ser comparados en función de su edad madurativa. Así, en las investigaciones más recientes se recomienda clasificar a los deportistas en maduradores tardíos, precoces o normomaduradores, o bien controlar el grado de maduración del deportista (e. g., Coelho e Silva, Figueiredo, Moreira y Malina, 2008; Coelho e Silva et al., 20Io; Goncalves, Rama y Figueiredo, 20I2; Matthyss et al., 2oII; Meylan, Cronin, Oliver y Hughes, 2oro), para poder eliminar la influencia de la maduración biológica sobre el resultado obtenido en los diferentes test utilizados en las baterías.

Toda esta situación se complica aún más si se observa la evolución que experimentan las especialidades deportivas a lo largo del tiempo, lo que hace aún más difícil dicha predicción. Los cambios reglamentarios producen modificaciones en las habilidades deportivas, lo que puede provocar que las variables o características que están midiendo las baterías de tests actuales ya no sean tan importantes o discriminantes en el futuro o que características muy importantes de la especialidad deportiva en el futuro aún no se tengan en cuenta (Vaeyens et al., 2008).

Por último, es preciso decir que, aún, muchos de los test que se utilizan no son pertinentes o no son capaces de valorar ciertas capacidades determinantes. Por ejemplo, en relación a los test de carácter técnico, es preciso observar que la mayoría de ellos se realizan en situaciones aisladas, siendo tareas normalmente cerradas, alejadas del contexto real, en las que la fatiga y factores de carácter externo como el tiempo o el resultado no influyen, siendo este aspecto una de las principales debilidades de este tipo de baterías (e. g., Karalejic, Jakovljevic y Macura, 20II). Este tipo de test tienen poca o ninguna correlación con las demandas que sufre el deportista durante la competición, especialmente en el caso de los juegos deportivos, que se caracterizan por una gran variabilidad y dinamismo espacio-temporal, donde los jugadores tienen que responder a los estímulos procedentes de sus compañeros, adversarios y el objeto de juego, provocando tal variabilidad que ninguna acción de juego dinámico se reproduzca dos veces de la misma manera (Phillips, Keith, Renshaw y Portus, 2010). Así pues, muchas veces tales test proporcionan mucha validez interna a la prueba, pero carecen de validez externa o ecológica.

Como consecuencia de todas estas limitaciones, es preciso considerar entonces las siguientes conclusiones a la hora de, bien diseñar baterías de test, o bien aplicarlas:

I. El perfil fisiológico, cineantropométrico, condicional y psicológico del jugador debe ser entendido como una forma de control del desarrollo del deportista, pero no como una herramienta de selección. De esta forma, se podrán conocer las fortalezas y debilidades del jugador y orientar el entrenamiento (Pearson, Naughton y Torode, 2006). La identificación de ciertos atributos sólo debe servir como una guía, más que como la inclusión de criterios de decisión en el 
desarrollo de los deportistas (Burgess y Naugthon, 20Io). Sin olvidar, además, la constante presencia del fenómeno de la compensación (Simonton, 1999), presente en todas las personas.

2. Es preciso incluir variables de tipo psicosocial en las baterías de test, ya que, a medida que aumenta la edad de los deportistas, las características físicas y fisiológicas son similares, mientras que las características psicológicas son consideradas como las más significativas (Vaeyens et al., 2008).

3. Las pruebas que dan mayor información son aquellas que implican realizarse en las mismas condiciones de la competición, incluyendo no sólo los gestos y las tomas de decisión, sino también la fatiga y otros condicionantes externos (Lidor, Côté y Hackfort, 2009). Al mismo tiempo, es preciso combinar este tipo de test con la observación del deportista en la competición a través de entrenadores expertos (Christensen, 2009).

4. Frente a los resultados obtenidos en las diferentes mediciones realizadas, y dadas las limitaciones existentes, el mejor indicador no es el resultado en sí del test, sino el porcentaje de aprendizaje del deportista, entendido como la velocidad en adquirir nuevas habilidades o la capacidad de mejora (Burgess y Naugthon, 2010; Vaeyens et al., 2008; Van Rossum y Gagné, 2005).

5. Es necesario el desarrollo de estudios de tipo longitudinal que permitan diferenciar entre el rendimiento actual del deportista y su potencial de rendimiento para, de esta manera, superar las limitaciones de los estudios transversales. Una alternativa práctica a esta limitación sería utilizar un proceso de confirmación del talento, entendido este como un periodo corto de tiempo, donde se enfrenta al deportista a situaciones de entrenamiento y competición, con el fin de valorar su adaptación al programa de desarrollo deportivo (Vaeyens et al., 2008).

\section{3. ¿Cómo es el camino para alcanzar el máximo rendimiento?}

Todas las limitaciones anteriormente citadas, así como la dificultad de objetivar el proceso de detección, más algunas otras consideraciones como puedan ser el abandono deportivo, la coordinación de la exigencia deportiva con otras actividades del deportista, la enorme oferta de actividad física existente en la actualidad y la consiguiente competencia entre las especialidades deportivas, la evolución social y la aparición de nuevas costumbres más sedentarias... (Lorenzo, 2003; Lorenzo y Sampaio, 2005) han provocado que muchos investigadores se planteen que en vez de utilizar una temprana $(D e)$ Selección, se ponga el énfasis en el desarrollo del deportista (Vaeyens et al., 2008). A partir de aquí, se pueden encontrar algunos factores claves que condicionen esos resultados o, incluso, algunas fases comunes en el desarrollo de este tipo de deportistas (una buena revisión y clasificación de los distintos factores que condicionan el entorno de proceso de desarrollo del talento se puede leer en $\mathrm{Li}$, John Wang y Young Pyun, 20I4).

Son dos fundamentalmente las investigaciones y los conceptos que han desencadenado esta línea de investigación. Por un lado, el concepto de práctica deliberada, 
desarrollado por Ericsson y colaboradores (e. g., Ericsson, Krampe y Tesch-Römer, 1993; Ericsson, 1996); y por otro lado, los estudios de Bloom (1985) y Côté y colaboradores (e. g., Côté, 1999; Côté y Hay, 2002), en los que encuentran etapas comunes en el desarrollo de los deportistas, y se observa como los distintos factores van evolucionando a lo largo de dichas etapas. Estos conceptos originales han auspiciado numerosas investigaciones que nos han permitido conocer algo más sobre la evolución del deportista, y han generado modelos de desarrollo del deportista que en la actualidad se encuentran vigentes (ver la revisión de Gulbin, Croser, Morley y Weissensteiner, 2013).

\section{I. ¿Todo depende de la práctica deliberada?}

La teoría de la práctica deliberada, propuesta por Ericsson, Krampe y TeschRömer (1993), ha servido como marco teórico para el estudio del desarrollo de los sujetos expertos durante los últimos años. La principal proposición de esta teoría es que el rendimiento de los sujetos expertos está directamente relacionado con la cantidad de horas de "práctica deliberada específica» acumuladas durante sus carreras profesionales. Para dichos autores, la práctica deliberada se define como una práctica altamente estructurada con el expreso deseo de progresar y mejorar y no con el deseo de pasarlo bien o entretenerse. Para Ericsson y cols. (en Williams y Franks, 1998: I62), la «teoría de la práctica deliberada sugiere que la natural habilidad no es un prerrequisito para desarrollar la pericia, sino que ésta depende más de la cantidad de tiempo gastado en una práctica "altamente estructurada" con un objetivo concreto de mejorar el rendimiento». Es importante destacar que, lejos de poner el énfasis en la cantidad de entrenamiento, esta teoría se centra en el tipo de entrenamiento utilizado por los sujetos.

Esta propuesta ha sido muy discutida en los últimos años, produciéndose diversas investigaciones alrededor de este aspecto. En general, se podría decir que, hasta la fecha, las investigaciones llevadas a cabo en distintos tipos de deportes (e. g., Baker, Côté y Abernethy, 2003a; Deakin y Cobley, 2003; Helsen, Starkes y Hodges, 1998; Hodge y Deakin, 1998; Hodges y Starkes, 1996; Starkes, Deakin, Allard, Hodges y Hayes, 1996; Ward, Hodges, Starkes y Williams, 2007; Williams y Hodges, 2005) querían confirmar los distintos principios planteados por Ericsson y colaboradores, y abrazaron sus argumentos con firmeza. La idea es básica y fácilmente aceptable: si quieres alcanzar el máximo rendimiento en cualquier ámbito de la vida, incluido el deporte, has de dedicarle tiempo, esfuerzo, estar concentrado, sacrificarte, trabajar de forma planificada... y los resultados llegaran antes o después. Y lo que los resultados de las investigaciones aportan es que:

a) Efectivamente, los deportistas expertos dedican más tiempo a su actividad que los novatos, aunque de una forma racional y equilibrada, alternando trabajo y descanso (deliberate recovery) (e. g., Baker, Côté y Abernethy, 2003a; Baker y Young, 20I4; Helsen et al., 1998; Hodge y Deakin, 1998; Starkes et al., 1996).

b) No sólo invierten más tiempo en el entrenamiento, sino que también dedican más tiempo a participar en las actividades específicas y más relacionadas con el rendimiento deportivo (Baker, Côté y Abernethy, 2003b; Baker y Horton, 2004); y 
c) A medida que aumenta la edad, las diferencias se van incrementando en cuanto al tiempo de práctica por diferentes motivos (e. g., mayor especialización de los más implicados o, por el contrario, posible abandono deportivo por no alcanzar las expectativas planteadas o cambio de orientación en la práctica deportiva...) (Starkes, 2000).

Sin embargo, con el paso del tiempo, y el desarrollo de las investigaciones en el ámbito deportivo, se ha profundizado más en el concepto y en sus implicaciones, así como han surgido distintas matizaciones y críticas a la teoría de la práctica deliberada (e. g., Abernethy, Farrow y Berry, 2003; Baker y Young, 20I4; Ruiz, Sánchez, Durán y Jiménez, 2006; Singer y Janelle, I999). Quizás, como primera crítica importante a esta teoría, es que aparentemente solo hace énfasis en los factores del tiempo empleado y la cantidad de práctica realizada. Está claro que para adquirir la excelencia en el deporte hace falta tiempo y práctica, pero estos factores no pueden ser los únicos en la adquisición de las habilidades deportivas (Baker y Davids, 2006), y se minimiza mucho la posible influencia de los factores genéticos (Tucker y Collins, 20I2). Por ejemplo, en un reciente estudio, Mossing, Madison, Pedersen, Kuja-Halkola y Ullén (20I4) analizaron la relación entre la práctica y la habilidad para tocar música en I0.500 gemelos. La conclusión fue que la habilidad para tocar música es más dependiente de los factores genéticos $(40-70 \%)$ que de la cantidad de práctica.

Por otro lado, es importante destacar que no todas las actividades realizadas en el ámbito deportivo coinciden plenamente con la definición original de práctica deliberada (en su origen, dicha teoría surge en un contexto diferente al del deporte, como fue el entorno de los violinistas), como puede ser el trabajo colectivo con un objetivo táctico o estratégico desarrollado por un equipo de fútbol. ¿Cuáles son las actividades que encajan en esa definición?, ¿el entrenamiento físico?, ¿el táctico?, ¿o el técnico?, ¿todos los tipos de entrenamiento? De esta forma, actividades no desarrolladas en solitario o bien actividades que son placenteras y divertidas no coinciden con el criterio original de la práctica deliberada (se recomienda leer el artículo de Baker y Young, 20I4). En este sentido, existen muchas actividades que son importantes para el desarrollo de la pericia y que no coinciden con el criterio original como puede ser la observación de otros deportistas, la participación en actividades de forma libre y recreativa o la participación en competiciones (Berry, Abernethy y Côté, 2008).

Algunas de las evidencias encontradas en este sentido en los deportes colectivos confirman que la participación en competiciones adquiere un papel fundamental en el desarrollo de la pericia de los deportistas (e. g., Baker, Côté y Abernethy, 2003a, 2003b; Starkes et al., 1996). La competición no se ajusta perfectamente al criterio establecido por Ericsson y colaboradores sobre práctica deliberada, ya que no se hace con el objetivo de mejorar, sino de competir y ganar. Sin embargo, en la competición se reúnen ciertos factores que son muy difíciles de conseguir en contextos de entrenamiento y, por tanto, debe ser considerada como un medio fundamental para el desarrollo de la pericia.

En los últimos años, han surgido investigaciones con el objetivo de poder cuantificar cuánto exactamente «la práctica deliberada» podía explicar o era la responsable del rendimiento obtenido por una persona. Así, por ejemplo, Hambrick et al. (2013), en el estudio que realizan para analizar la influencia de la práctica deliberada en jugadores expertos de ajedrez y músicos, concluyen que ésta tan solo explica el $34 \%$ de 
la varianza del rendimiento en el caso de los jugadores de ajedrez, y en el caso de los músicos tan solo explica el 30\%. Dados estos resultados, estos autores señalan la necesidad de analizar la influencia de otros factores que expliquen el rendimiento como son la edad de comienzo, la personalidad o el nivel de inteligencia.

Más recientemente, Macnamara, Hambrick y Oswald (20I4) han realizado un metaanálisis sobre la influencia de la práctica deliberada en distintos dominios como son la música, los deportes, la educación o el ámbito profesional. Trataron de responder a la pregunta de cuánto de la variación total del rendimiento puede ser explicada a partir de la cantidad de práctica deliberada. Los resultados no pueden ser más evidentes: la práctica deliberada tan solo explica el ı2\% de la variación del rendimiento $(26 \%$ en el caso de los juegos, $21 \%$ en el caso de la música, $18 \%$ en el caso de los deportes, $4 \%$ en el caso de la educación y tan solo un $\mathrm{i} \%$ en el caso de las profesiones). Es decir, que una gran cantidad del rendimiento no es explicado por la práctica deliberada y sí por otros factores (por ejemplo, la edad de comienzo de la práctica o las diferencias individuales).

En el mismo estudio, también se observa qué relación existe entre la predictibilidad de las tareas (es decir, una tarea muy predecible es correr Ioo metros, mientras que una tarea poco predecible puede ser cuándo surge un accidente de motos en una carrera del Gran Premio) y la influencia de la práctica deliberada en el rendimiento. En el caso de las tareas más predecibles, más estables, el porcentaje del rendimiento explicado por la práctica deliberada es mayor, siendo considerablemente menor en tareas de muy poca estabilidad.

3.2. ¿Necesita ser la práctica, el entrenamiento, exclusivamente deliberado? ¿ Existen otras formas de entrenamiento también adecuadas para obtener rendimiento?

Del mismo modo, las distintas investigaciones realizadas han tratado de conocer no sólo si la diferencia entre los deportistas expertos y los no expertos se debía al tipo de entrenamiento realizado, sino también a cómo han evolucionado las distintas actividades que podían realizar los deportistas, si han practicado varios deportes o no antes de especializarse en uno concreto, o si, además de la práctica deliberada, han utilizado otro tipo de práctica con diferentes características (e. g., Baker et al., 2003b; Baker y Horton, 2004; Deakin y Cobley, 2003). Por ello, además de la práctica deliberada como un factor que contribuye al desarrollo de la pericia, Côté y colaboradores (Côté y Hay, 2002; Côté, Baker y Abernethy, 2003) han propuesto un concepto diferente, que también contribuye al desarrollo de la pericia, que es el término de «juego deliberado» (deliberate play). Según Côté (1999) y Côté y Hay (2002), la estructura y los contenidos de los entrenamientos y juegos que realiza el deportista van evolucionando a lo largo de su desarrollo. De esta forma, seguro que las actividades consideradas más adecuadas en los últimos estadios de desarrollo de los jugadores no tienen nada que ver en cuanto a las actividades, e incluso en cuanto al entorno motivacional, que deben realizar los niños en sus primeros estadios de iniciación deportiva.

Por «juego deliberado» se entiende a la actividad que caracteriza fundamentalmente a los deportistas durante los primeros años, hasta aproximadamente los I2 años. Este tipo de actividades produce una gratificación inmediata, son motivantes, 
y están diseñadas para provocar la mayor diversión posible. El juego deliberado incluye actividades como el clásico partido de fútbol o baloncesto con los amigos en el parque, que son normalmente realizados con un menor número de jugadores y con adaptaciones reglamentarias. Este tipo de actividad se ha demostrado que contribuye a la formación y desarrollo de la inteligencia táctica y de la creatividad táctica (e. g., Bell-Walker y Williams, 2008; Greco, Memmert y Morales, 20Io), y que incluso tiene una influencia positiva en la motivación y compromiso del deportista con la misma actividad (Côté, Baker y Abernethy, 2007). Otra de las grandes ventajas que aporta este tipo de práctica es que el tiempo útil de trabajo, el tiempo útil en el que el niño está implicado en el deporte es mucho más alto en comparación con otro tipo de actividades más reguladas, como la práctica deliberada, en el que otros factores como las explicaciones del entrenador, la organización de los ejercicios, etc., reducen el tiempo útil de trabajo (Baker y Young, 20I4).

Resultado de las distintas investigaciones desarrolladas, se sugiere que ambos tipos de práctica, práctica deliberada y juego deliberado, contribuyen de forma significativa al desarrollo de la pericia, especialmente en los deportes donde la percepción y la toma de decisión tienen un papel prioritario (Baker et al., 2003b; Berry et al., 2008; Soberlak y Côté, 2003). Por ejemplo, Berry et al. (2008) analizaron la influencia de la práctica deliberada y del juego deliberado en el desarrollo de la capacidad de percepción y de toma de decisión en jugadores de la liga de fútbol australiano (jugadores del mismo nivel competitivo, pero diferenciados en función de su capacidad en la toma de decisión). Sus resultados mostraron que no había diferencias entre ambos tipos de jugadores en el número de actividades estructuradas, aunque los jugadores expertos al llegar a la liga tenían más horas acumuladas de prácticas estructuradas. Del mismo modo, los deportistas expertos tenían más horas de actividades estructuradas en deportes de invasión, siendo éste un factor que discriminaba a ambos tipos de jugadores, así como también se encontraron diferencias significativas en las horas invertidas en actividades de juego libre en deportes de invasión.

Del mismo modo, Memmert, Baker y Bertsch (20I0), con el objetivo de conocer qué favorecía el desarrollo de la creatividad táctica en los deportes de equipo, aplicaron un cuestionario a 72 jugadores profesionales de baloncesto, fútbol, balonmano y hockey hierba, divididos en dos grupos: jugadores más creativos y menos creativos. El principal resultado encontrado fue que los jugadores más creativos en cada deporte habían dedicado más tiempo a ambos tipos de práctica, encontrándose diferencias significativas tanto en el juego libre como en la práctica deliberada. En los primeros años ( 5 a I4 años) estas diferencias sólo se encontraron en el juego libre.

Basándose en estas investigaciones, Côté et al. (2007) propusieron un Modelo de Desarrollo Deportivo (DMSP), en el que fundamentalmente se pueden distinguir dos caminos diferentes de desarrollo de la pericia deportiva: la temprana diversificación o la especialización temprana. Una reciente revisión de dicho modelo y sus implicaciones se puede encontrar en el trabajo de Côté y Vierimaa (20I4).

En la temprana diversificación, que está influenciada por los estudios de Bloom (1985), se observa que se produce una evolución en el deportista, desde el juego deliberado hasta la práctica deliberada, y desde la diversidad hacia la especificidad. En el caso contrario, en la especialización temprana, los deportistas comienzan en un deporte a edad temprana y dedican mucho tiempo a un entrenamiento específico, práctica deliberada, de dicho deporte. Este debate continúa en la actualidad de forma muy viva, 
aportando ambas visiones argumentos a favor de una y otra (una interesante argumentación de esta polémica se puede leer en Côté, Lidor y Hackfort, 2009), y analizando consecuencias e implicaciones de las diferentes trayectorias deportivas (e. g., Strachan, Côté y Deakin, 2009). Tratando de resumir, tenemos que pensar que, como bien señalan Ford y Williams (20II), ambos tipos de desarrollo no deben ser entendidos dentro de un modelo dicotómico, o la opción de la especialización temprana o la opción de la diversidad. De hecho, muchas de las variables descritas en dicha trayectoria deportiva (número de actividades deportivas practicadas, horas dedicadas al deporte principal, etc.) forman parte de un contínuum que evoluciona y pueden coexistir a la vez (practicar varios deportes y dedicar muchas horas a una actividad deportiva).

Paralelamente al debate sobre la práctica deliberada y el juego deliberado, existe un segundo debate relacionado con la práctica exclusivamente en el deporte elegido, o bien practicar varios deportes antes de especializarse en un solo deporte (e. g., ver la revisión de Côté et al., 2009; Côté et al., 2007; Güllich y Emrich, 20I2). Son varias las investigaciones realizadas analizando la trayectoria de los deportistas, y los resultados obtenidos hasta la fecha son inconsistentes. Quizás uno de los estudios más fiables realizados hasta la fecha sea el de Güllich y Emrich (2012), fundamentalmente por el tamaño de la muestra utilizada (I566 deportistas alemanes, diferenciándolos entre deportistas de nivel mundial -olímpicos o participación en campeonatos del mundo- y deportistas de nivel nacional -que estaban en el top ten del ranking nacional-). Los resultados mostraron que, no habiendo diferencias entre ambos grupos en el volumen y la intensidad total del entrenamiento, se observaron diferencias en que la proporción de deportistas de nivel internacional que habían practicado varios deportes era mayor que en los deportistas de nivel nacional. Es decir, que aunque no existían diferencias en el volumen de entrenamiento específico en el deporte principal, los deportistas de nivel internacional habían practicado y competido en más deportes durante la infancia y adolescencia, habiéndose especializado más tarde. Finalmente, en este estudio, también se concluyó que la mayoría de los deportes practicados por los deportistas eran deportes relacionados con el deporte principal.

En la misma línea, Zibung y Conzelmann (20I2) analizaron la trayectoria deportiva de jóvenes jugadores de fútbol a partir de la interacción de cuatro factores (horas de entrenamiento específico en fútbol, horas de juego libre en fútbol, otros deportes practicados y edad de comienzo del entrenamiento específico). A partir de su estudio, pudieron identificar diferentes modelos de trayectorias deportivas, destacando dos que habían permitido alcanzar el máximo rendimiento deportivo. Una de ellas, lo que llamaron «jugadores especializados», se caracterizaba por una elevada práctica específica o deliberada, por una elevada cantidad de juego deliberado y poca participación en otros deportes. La otra trayectoria que permitió alcanzar el alto rendimiento en fútbol (nivel internacional, «jugadores polideportivos»), se caracterizó por una elevada cantidad de práctica deliberada (por encima de la media), una baja cantidad de juego de juego deliberado pero sí mayor participación en más deportes. La explicación a este hecho, por parte de los autores, es que una cantidad de juego deliberado puede ser sustituido por la participación en otros deportes. Sin embargo, tal y como señalan los propios autores, la trayectoria al máximo nivel en fútbol viene condicionada por tratarse de un deporte muy popular, con una gran densidad de jugadores y de competición. Es decir, esa posible especialización necesaria está condicionada por aspectos culturales, como ya hemos comentado anteriormente. 
Otro trabajo similar es el presentado recientemente por Güllich (20I4), sobre las diferentes trayectorias deportivas seguidas por jugadores de hockey hierba alemanes campeones olímpicos en Londres 20I2, y además comparan dicha trayectoria por la seguida por otros jugadores de nivel nacional o por jugadores internacionales y medallistas una década anterior. Los resultados muestran que los jugadores olímpicos presentan un nivel medio de práctica deliberada, pero muestran una mayor implicación en otros deportes y que su especialización es más tardía, siendo estos dos últimos datos lo que realmente les diferencian de los jugadores de nivel nacional... Conclusión: Todos los caminos conducen a Roma.

Como conclusiones a este debate, es preciso considerar que: a) tanto la práctica deliberada como el juego libre tienen un papel crucial en el desarrollo de la creatividad; b) que el juego libre es importante hasta un momento concreto, en el que ya no es suficiente; c) que, el juego libre y la mayor cantidad de experiencias parecen favorecer el desarrollo de la creatividad táctica; y d) que, de forma bastante consistente, se empiezan a argumentar razones de tipo contextual y cultural para explicar las diferencias encontradas en los distintos estudios. Es posible, en este sentido, que los volúmenes de entrenamiento o el tipo de práctica se puedan ver afectados por las distintas políticas deportivas de los países o las distintas especialidades deportivas (por ejemplo, diferencias entre deportes individuales y colectivos). Del mismo modo, y siendo una importante conclusión de esta línea de investigación, se encuentra la variabilidad individual de cada deportista. Es decir, que el mismo tipo de actividades puede conllevar diferentes rendimientos y, al revés, diferentes patrones de entrenamiento pueden llevar al mismo nivel de rendimiento (Güllich, 20I4).

De esta forma, se entiende que son varios los factores que pueden condicionar el desarrollo de una trayectoria u otra: el tipo de deporte (deportes de rendimiento precoz o deportes de rendimiento tardío; deportes colectivos o individuales); la cultura deportiva del país (deportes que tienen un reconocido impacto social en dicho país, como puede ser el fútbol en Inglaterra, el hockey sobre hielo en Canadá o el rugby en Nueva Zelanda -imagínense a qué deporte se puede dedicar alguien cuando vive en un país en el que un partido de la Copa del Mundo de rugby consigue una cuota de pantalla en televisión del 80\%-) o la profundidad de la competición pueden explicar por qué se producen esas variaciones en el desarrollo de la pericia de los deportistas. No obstante, aún se considera necesario realizar más investigaciones, que consigan superar ciertas limitaciones metodológicas (e. g., clasificación de los sujetos) y que consigan superar ciertas limitaciones culturales, que impliquen análisis transnacionales, como sugieren Stambulova y Alfermann (2009).

\section{3. ¿El resultado, el rendimiento solo pasa por dedicarle mucho tiempo?...}

De acuerdo con Vaeyens, Güllich, Warr y Philippaerts (2009), la mayoría de los programas de detección y desarrollo del talento están preocupados por la identificación y el reclutamiento de deportistas talentosos en una edad temprana, para, de esta manera, implicarles en la actividad deportiva durante un largo periodo de tiempo antes de alcanzar los resultados deportivos. Para dichos autores, esta tradicional aproximación está basada en razones del tipo: a) el éxito a nivel internacional es el resultado de una larga implicación en una sola disciplina deportiva; b) el éxito aumenta con la 
duración del entrenamiento y la competición en el deporte elegido; o c) una temprana implicación, el éxito temprano y la continuación en programas de promoción deportivos estimularán el proceso de desarrollo del talento y, por tanto, correlaciona positivamente con el éxito en el alto rendimiento deportivo.

Sin embargo, si analizamos diversas investigaciones veremos que esto no es del todo cierto.

Primera evidencia: No es real que haya que permanecer mucho tiempo implicado en un programa de detección de talentos para obtener rendimiento

Diversos estudios e investigaciones cuestionan dicha orientación. Por ejemplo, Gullich (2013) analizó el programa de detección y promoción del talento en fútbol, en Alemania. El objetivo fundamental de su investigación es conocer si realmente dicho programa de promoción del talento está basado en un proceso temprano de identificación o, si por el contrario, se sustenta sobre un proceso constante de selección y deselección de los jugadores en las diferentes categorías, en cuyo sistema los jugadores entran y salen, cambiando a lo largo de todo el proceso. Para ello, analizaron todo el programa alemán durante las temporadas 200I a 2013. Los resultados demuestran que la media de cambio de jugadores era del $24.5 \%$ en los centros de jóvenes jugadores, mientras que en los equipos nacionales era del $4 \mathrm{r} \%$. En cualquier categoría, la probabilidad de permanecer en el programa tres años después era menor del $50 \%$.

Lo que viene a confirmarnos este estudio es que, realmente, esa idea de empezar pronto en un programa de detección de talentos y permanecer largo tiempo en el, con el compromiso de mejorar, no es realmente cierta. La realidad es que este tipo de programas se van alimentando a lo largo del tiempo de diferentes deportistas y van cambiando; estos programas presentan una gran fluctuación en los deportistas.

Parece que una temprana participación en competiciones y la inclusión en programas de detección y desarrollo del talento correlacionan negativamente con el éxito (Güllich y Emrich, 20I2). Güllich y Emrich (2006a) analizaron el historial deportivo de los deportistas alemanes que participaron en las olimpiadas, tratando de encontrar que relación había entre la participación en programas de detección y promoción deportiva y el éxito alcanzado posteriormente. Entre otros resultados encontraron que los deportistas internacionales empezaron el entrenamiento y la competición (nacional e internacional) significativamente más tarde que otros menos exitosos (solamente competían a nivel nacional). Además, una gran proporción de dichos deportistas internacionales habían entrenado y competido en otros deportes diferentes a su principal deporte. Consecuencia de ello, estos sujetos eran seleccionados por las respectivas federaciones a una edad mucho más tardía. Muy probablemente, por debajo de esta casuística, se encuentre un argumento anteriormente ya comentado, como es el hecho de la influencia que ejerce la maduración biológica del deportista en el rendimiento deportivo.

En resumen, el análisis de la eficacia de los diferentes sistemas de promoción deportiva revela una baja o moderada proporción de éxito. Parece que la mayoría de los deportistas reclutados en edades tempranas nunca llegan a ser deportistas de éxito a nivel sénior. Por otro lado, muchos de los deportistas internacionales no han formado parte de programas deportivos institucionales. 
Segunda evidencia: La edad de comienzo de la práctica deportiva varía y se puede empezar tarde a practicar un deporte

Tal y como afirman Moesch, Trier, Wikman y Elbe (2013), una lógica conclusión de la teoría de la práctica deliberada es que es necesaria una edad temprana en el comienzo de la actividad deportiva, para de esta forma alcanzar un grado de pericia suficiente en relación a otros deportistas que también practican dicha especialidad deportiva (no olvidemos, en este sentido, que el concepto de talento es un concepto social, que necesita de la comparación para su reconocimiento).

Sin embargo, son varias las investigaciones que han demostrado que la edad de iniciación en el entrenamiento y la competición varía de forma muy evidente entre deportistas exitosos. Güllich (2007) analizó los datos de 4455 deportistas olímpicos que participaron en las Olimpiadas de Atenas (2004), sobre la edad de comienzo a entrenar en su especialidad deportiva. Los resultados sugieren que la edad de comienzo del entrenamiento específico varía entre y dentro de la misma disciplina deportiva, y que un considerable porcentaje de deportistas internacionales comienzan a entrenar después del tradicional «timing» de la identificación del talento (aproximadamente entre los 8-I2 años), lo que implica que para obtener el éxito en algunos deportes olímpicos no es necesario comenzar a una edad muy temprana el entrenamiento específico.

Es más, tal y como veremos más adelante, es posible cambiarse de deporte a una edad tardía y tener éxito. Serán varias las cuestiones que favorezcan dicha circunstancia como el hecho de pasarse a un deporte con una menor profundidad competitiva, o una menor densidad de jugadores, y donde obtener rendimiento sea algo más factible.

Tercera evidencia: No es necesario empezar a competir pronto en la especialidad deportiva

Muy unido al anterior argumento, ya hemos comentado el hecho de que los atributos necesarios para alcanzar el máximo rendimiento no aparecen al mismo tiempo, y que pueden surgir en edades tardías. O que, dada la evolución de las especialidades deportivas, algunas características necesarias aún no se conozcan, mientras que las nuevas metodologías de entrenamiento, las modificaciones reglamentarias o la aplicación de las nuevas tecnologías provoquen diversos tipos de deportistas.

Entre los deportistas olímpicos de Atenas 2004 (Güllich, 2007), tan solo el 44\% de ellos habían debutado en competición internacional en su disciplina deportiva en la categoría júnior ( $6.8 \pm 2.5$ años). La mayoría de ellos habían competido a nivel internacional por primera vez en la categoría sénior (22.0 \pm 3.I años). En el estudio realizado por Schumacher, Mroz, Mueller, Schmid y Ruecker (2006), sobre la carrera deportiva seguida por los ciclistas profesionales, tan solo el $29.4 \%$ de ellos habían participado en campeonatos del mundo júnior, mientras que solo el $34 \%$ de aquellos que habían competido en dichos campeonatos mundiales habían tomado parte después en las principales competiciones sénior. Recientemente, Barreiros, Côté y Fonseca (2012) trataron de analizar la participación de 395 deportistas portugueses, de diferentes deportes, con el objetivo de conocer cuántos deportistas que en categorías inferiores habían sido internacionales lo eran en categoría sénior. Los 
resultados encontrados confirmaron que tan solo un tercio de los deportistas que son internacionales en categoría sénior han competido anteriormente a nivel internacional en categorías inferiores.

Más aún, también debemos pensar que la trayectoria del deportista a nivel competitivo no es una constante, sino que presenta una gran variabilidad no solo en cuanto a la edad de comienzo, sino incluso también en cuanto a la progresión por las distintas categorías y niveles competitivos. En el estudio de Gulbin, Weissensteiner, Oldenziel y Gagné (2013), el 83\% de los deportistas encuestados presentaron una trayectoria competitiva no lineal. Estos resultados demuestran que, frente a la tradicional visión del desarrollo del talento en forma piramidal, es muy difícil encontrar un desarrollo lineal, y que la trayectoria deportiva se encuentra afectada por numerosas oscilaciones.

Cuarta evidencia: No es necesario dedicar mucho tiempo

Una de las ideas más aceptadas durante muchos años hace referencia a la cantidad de tiempo necesaria de práctica deliberada para alcanzar el máximo rendimiento. Así, rápidamente se popularizó la regla de los io años, y posteriormente, la regla de las Io.000 horas de práctica deliberada. Esta idea surgió del estudio de Ericsson et al. (1993), en el que los músicos expertos habían tenido un total de I0.00o horas de práctica deliberada a la edad de los 20 años, mientras que los músicos menos expertos tan solo habían acumulado un total de 2.000 de dicha práctica.

Desde entonces, son varios los estudios que han intentado encontrar la relación entre la cantidad de práctica y el rendimiento obtenido, encontrándose resultados poco concluyentes y contradictorios (e. g. Baker et al., 2003a; Helsen et al., I998; Starkes et al., 1996). A la vista de los distintos resultados, no resulta tan evidente que se necesite una larga implicación en un deporte para obtener rendimiento. Esa clásica idea de la regla de los io años o las ı..ooo horas no es totalmente cierta (Baker y Young, 20I4).

Por ejemplo, Gulbin y colaboradores (Gulbin, 2006; Oldenziel, Gagné y Gulbin, 2004), investigaron sobre la trayectoria deportiva de deportistas australianos de alto rendimiento a través de cuestionarios. De los 459 participantes que le respondieron, y que habían representado a Australia en competiciones internacionales tanto en edad júnior como sénior, la duración media del periodo transcurrido entre el primer contacto con el deporte y la primera competición internacional fue de $7.5 \pm 4$.I años. El $70 \%$ de los deportistas habían requerido menos de io años para alcanzar la pericia. En comparación con aquellos deportistas que habían alcanzado la excelencia (entendida ésta como haber competido a nivel internacional) después de ro años o más, los «quick-developers» se caracterizaban por haber llegado al deporte relativamente tarde (a los I7.I \pm 4.5 años), por haber experimentado una gran variedad de deportes antes de la especialización, por comenzar en niveles competitivos elevados y por participar en competiciones júnior y sénior al mismo tiempo.

Güllich (20I4) afirma lo siguiente: «No solo la cantidad de entrenamiento no supone el éxito, sino que además el volumen exigido para ser un deportista internacional está muy por debajo de las I0.00o horas propuestas por Ericsson et al.». 
Quinta evidencia: Es posible practicar varios deportes y luego obtener rendimiento en uno

En otro sentido, muchos deportistas internacionales no han progresado a través de una carrera deportiva lineal, es decir, dentro de una sola especialidad deportiva, sino que han practicado numerosos deportes durante la infancia y la adolescencia (e. g., Leite, Baker y Sampaio, 2009). De los estudios realizados en este ámbito parece deducirse que de los dos grupos de deportistas, de larga implicación y de corta implicación en una sola disciplina deportiva, estos últimos han comenzado dicha especialización a una edad más tardía y que se han involucrado en varios deportes antes.

Güllich y Emrich (2006b), en otro estudio en el que analizaron de forma longitudinal la actividad deportiva de I2I deportistas olímpicos, encontraron que un mayor volumen de entrenamiento en otros deportes junto con un menor volumen de implicación en programas institucionales en 1999 estaba asociado con mayor éxito en el año 2002. Por el contrario, el volumen de entrenamiento en la propia especialidad deportiva en el año 1999 no explicaba significativamente el éxito en el 2002, mientras que una gran proporción del total del entrenamiento hasta el año 1999 en la modalidad deportiva se correlacionaba con menos éxito en el 2002. Güllich (2014) confirma que los jugadores de hockey que fueron medalla de oro en las Olimpiadas de Londres (20I2) empezaron su implicación en la actividad deportiva a una edad temprana, presentaron niveles moderados de entrenamiento estructurado de su especialidad deportiva en comparación con otros jugadores de menor nivel competitivo, pero que practicaron varios deportes y que se especializaron más tarde.

Más aún, existen claros ejemplos de deportistas olímpicos que han sido capaces de cambiar de un deporte a otro compitiendo en los dos a nivel internacional (Gulbin, 2008). Yelena Isinbaeva cambió de deporte a los is años, y ha sido capaz de ganar el oro en dos olimpiadas. Después de ganar dos medallas de bronce en ciclismo, Clara Hughes se cambió de deporte, ganando tres medallas, incluida una de oro, en patinaje de velocidad en los juegos olímpicos.

Estas situaciones han provocado la aparición de nuevas alternativas para encontrar y desarrollar deportistas de nivel internacional. Entre ellas se pueden destacar dos fundamentalmente: la «identificación de talentos maduros» («mature-age talent identification») y el «reciclaje de talentos» («talent recycling») (Vaeyens et al., 2009) o «transferencia de talentos» («transfer talent», Burgess y Naugthon, 20ıо; Reilly et al., 20oob). Estos conceptos, lejos de ser una propuesta teórica, son una realidad que en la actualidad se han aplicado en distintos programas para conseguir obtener rendimiento («Sporting Giants», «Pitch2Podium», «Girls4Gold»...) (para tener un mayor conocimiento de estos programas se recomienda ver la pagina de UK Sport, el apartado de «Frontiers Solutions», dentro de los programas de detección de talentos, o bien leer Lorenzo et al., 20I4). Sin embargo, tal y como apuntan Collins, Collins, Macnamara y Jones (20I4), estos procesos (pese a representar el 7.5\% de los deportistas olímpicos de los equipos de Estados Unidos, Gran Bretaña y Canadá), normalmente se producen de forma espontánea y no están nada claros aún los mecanismos que explican dichos procesos de transferencia de un deporte a otro y por qué se obtiene éxito o no.

De estos datos se puede deducir que: a) no es necesario estar incluido en un programa de detección y desarrollo del talento desde una temprana edad; y b) que es 
posible cambiar de disciplina deportiva a una edad relativamente tardía y alcanzar resultados deportivos.

\section{Conclusiones}

Son numerosos los interrogantes que aún quedan sin responder sobre el proceso de detección y desarrollo del talento. Desde un punto de vista conceptual o desde un punto de vista práctico, aún son necesarias más investigaciones en este ámbito para conocer realmente cómo se produce el proceso formativo de un deportista y cómo se pueden desarrollar nuevas estrategias formativas, permitiendo el desarrollo tanto del deportista como de la persona. Los cambios sociales y los cambios experimentados por las propias especialidades deportivas obligan, en este sentido, a replantear constantemente el proceso.

A lo largo de las investigaciones realizadas hasta la actualidad son muchos los factores que condicionan dicho proceso. Al mismo tiempo, son varios los condicionantes que van surgiendo y que provocan que el proceso formativo de un deportista sea algo absolutamente específico a cada deporte y a cada cultura. En este sentido, no sólo se necesitan más investigaciones, sino que son necesarios estudios de tipo longitudinal y transnacionales o transculturales.

Finalmente, es preciso asumir cuanto antes que parece improbable que exista un único camino óptimo para alcanzar el máximo rendimiento deportivo en cada especialidad deportiva. Las características biológicas de cada individuo y el efecto de las interacciones que se producen con su entorno provocan que cada deportista genere diferentes tipos de soluciones, dependiendo de sus características y de la relación exponencial que tenga con las múltiples variables del rendimiento. Y que diferentes variables de entrenamiento puedan ser efectivas, en función de cómo cada deportista diseñe su progreso durante muchos años, de la forma que sea la más adecuada a su desarrollo individual, gestionando su nivel de tolerancia al entrenamiento y competición al mismo tiempo que es capaz de coordinar su actividad deportiva con otros intereses fuera del deporte, como puedan ser la familia, los amigos, la educación, etc.

Por ello, siendo uno de los factores claves la necesidad de asegurar que el deportista permanezca implicado con la especialidad deportiva durante un tiempo, será preciso desarrollar o crear entornos de entrenamiento adecuados que comprometan al deportista y que incentiven el aprendizaje así como la autogestión.

\section{Bibliografía}

Аввотт, A. y Collins, D. (2004) Eliminating the dichotomy between theory and practice in talent identification and development: considering the role of psychology. Journal of Sports Sciences, 22, 395-408. http://dx.doi.org/10.1080/02640410410001675324

Abernethy, B.; Farrow, D. y Berry, J. (2003) Constraints and issues in the development of a general theory: A critique of the deliberate practique framework. En J. L. STARKES y K. A. ERICsson (eds.) Expert performance in sports Champaign, IL: Human Kinetics, (pp. 89-IIо). 
Baker, J.; Côté, J. y Abernethy, B. (2003a) Sport specific practice and the development of expert decision-making in team ball sports. Journal of Applied Sport Psychology, I5, I2-25. http://dx.doi.org/I0.1080/10413200305400

BAker, J.; Côté, J. y Abernethy, B. (2003b) Learning from the experts: Practice activities of expert decision-makers in sport. Research Quarterly for Exercise and Sport, 74 (3), 342-347. http://dx.doi.org/I0.1080/02701367.2003.I0609IoI

BAKER, J. y DAVIDS, K. (2006) Genetic and enviromental constraints on variability in sport performance. En K. Davids, S. Bennett y K. Newell (eds.) Movement System Variability (pp. I09-I29). Champaign, IL: Human Kinetics.

BAKER, J. y HORTON, S. (2004) A review of primary and secondary influences on sport expertise. High Ability Studies, I5 (2), 21I-226. http://dx.doi.org/I0.1080/1359813042000314781

BAKER, J. y YOUNG, B. (20I4) 20 years later: deliberate practice and the development of expertise in sport. International Review of Sport and Exercise Psychology, 7: I, I35-I57. http://dx.doi.org/10.1080/1750984X.2014.896024

Barreiros, A.; Côté, J. y FonsecA, A. M. (2OI2) From early to adult sport success: Analysing athletes' progression in national squads. European Journal of Sport Science. http://dx.doi.org/I0.1080/17461391.20I2.671368

Bell-Walker, J. y Williams, A. M. (2008) The effect of memory recall on perceptual cognitive skill in elite soccer: development of long term working memory. En T. REILLY y F. KORKUSUZ (eds.) Science and football VI. London: Routledge.

Berry, J.; Abernethy, B. y Côté, J. (2008) The contribution of structures activity and deliberate play to the development of expert perceptual and decision-making skill. Journal of Sport E Exercise Psychology, 30, 685-708.

BLoom, B. S. (1985) Developing talent for young people. New York: Ballantine.

Bourgois, J.; Claessens, A. L. y Vrijens, J. et al. (2000) Anthropometric characteristics ofelite male junior rowers. British Journal of Sports Medicine, 34, 213-217. http://dx.doi.org/Io.II36/bjsm.34.3.213

Burgess, D. J. y Naugthon, G. A. (20ro) Talent development in adolescent team sports: A review. International Journal of Sports Physiology and Performance, 5, I03-II6.

Christensen, M. K. (2009) «An eye for talent»: Talent identification and the "práctical sense» of top-level soccer coaches. Sociology of Sport Journal, 26, 365-382.

Coelho e Silva, M. J.; Figueiredo, A. J.; Moreira, H. y Malina, R. M. (2008) Functional capacities and sport-specific skills of 14 to is-year-old male basketball players: Size and maturity effects. European Journal of Sport Science, 8 (5), 277-285. http://dx.doi.org/I0.1080/17461390802117177

Coelho e Silva, M. J.; Moreira, H.; Gonçalves, C. E.; Figueiredo, A. J.; Elferink-GemSer, M. T.; Phillippaerts, R. M. y Malina, R. M. (20Io) Growth, maturation, functional capacities and sport-specific skills in I2-I3 years-old-basketball players. Journal of Sports Medicine and Physicall Fitness, 50, 174-18I.

Collins, R.; Collins, D.; Macnamara, A. y Jones, I. M. (20I4) Change of plans: an evaluation of the effectiveness and underlying mechanisms of successful talent transfer. Journal of Sports Sciences. http://dx.doi.org/10.1080/026404I4.2014.908324

CÔTÉ, J. (1999) The influence of the family in the development of talent in sport. The sport psychologist, 13 , 395-417.

Cote, J.; BAKer, J. y Abernethy, B. (2003) From play to practice: a developmental framework for the acquisition of expertise in team sports. En J. L. STARKes y K. A. ERICsson (eds.) Expert performance in sports: advances in research on sport expertise (pp. 85-87; 89-II3; 4I44I6). Champaign, Ill.: Human Kinetics, c2003. United States. 
Côté, J.; Baker, J. y Abernethy, B. (2007) Play and practice in the development of sport expertise. En G. Tenenbaum y R.C. EkLund (eds.) Handbook of sport psychology (pp. I84202). Hoboken, NJ: John Wiley y Sons.

Côté, J. y HAY, J. (2002) Childrens involvement in sport: A developmental perspective. En J. M. Silva y D. Stevens (eds.) Psychological foundations of sport (pp. 484-502). Boston: Merrill.

Côté, J.; Lidor, R. y Hackfort, D. (2009) issp Position Stand: To Sample or to Specialize? Seven Postulates about youth sport activities that leads to continue participation and elite performance. International Journal of Sport and Excercise Psychology, 9, 7-17. http://dx.doi.org/10.1080/1612197X.2009.9671889

Côté, J. y VierimaA, M. (20I4) The development model of sport participation: is years after its first conceptualization. Science Sport. http://dx.doi.org/ıo.IoI6/j.scispo.20I4.08.133

Deakin, J. M. y Cobley, S. (2003) An examination of the practice environments in figure skating and volleyball: a search for deliberate practice. En J. STARKES y K. A. ERICSSON (eds.) Expert performance in sports: advances in research on sport expertise. Champaign: Human Kinetics.

Durand-Bush, N. y Salmela, J. (2002) The development and maintenance of expert athletic performance: Perceptions of world and olympic champions. Journal of Applied Sport Psychology, 2002, I4 (3), 154-171. http://dx.doi.org/I0.IO80/10413200290103473

Elferink-Gemser, M. T.; Visscher, C.; Lemmink, K. A. P. M. y Mulder, T. (2007) Multidimensional performance characteristics and standard of performance in talented youth field hockey players: A longitudinal study. Journal of Sports Sciences, 25 (4), 48I-489. http://dx.doi.org/I0.1080/02640410600719945

ENGLish InSTITUTE OF SPORT (20IO) Talent Identification. Recuperado el 6 de agosto de 20II, de http://www.eis2win.co.uk/pages/Talent_Identification.aspx.

ERICSSON, K. A. (1996) The road to excellence: The acquisition of expert performance in the arts and sciences, sports and games. Hillsdale: Lawrence Erlbaum.

Ericsson, K. A.; Krampe, R. y Tesch-Römer, C. (1993) The role of deliberate practice in the acquisition of expert performance. Phychological review, I00 (39), 363-406. http://dx.doi.org/Io.1037/0033-295x.100.3.363

FALK, B.; Lidor, R.; LANDER, Y. y LANG, B. (2004) Talent identification and early development of elite water-polo players: a 2-year follow-up study. Journal of Sports Sciences, 22 (4), 347-355. http://dx.doi.org/I0.I080/0264041031000I641566

Ford, P. R. y Williams, A. M. (20II) The developmental activities engaged in by elite youth soccer players who progressed to professional status compared to those who did not. Psychology of Sport and Exercise, $\mathrm{I}-4$.

Gabbett, T. J.; Jenkins, D. G. y Abernethy, B. (20II) Relative importance of physiological, anthropometric, and skill qualities to team selection in professional rugby league. Journal of Sports Sciences, 29 (13), I453-166r. http://dx.doi.org/Io.1080/026404I4.201I.603348

Gilar, R. y CASTEJÓN, J. L. (2003) El desarrollo de la competencia experta. Implicaciones para la enseñanza. Alicante: Editorial Club Universitario.

Gonçalves, C. E.; Rama, L. M. L. y Figueiredo, A. B. (20I2) Talent Identification and Specialization in Sport: An overview of some unanswered questions. International Journal of Sports Psychology and Performance, 7, 390-393.

Greco, P.; Memmert, D. y Morales, J. C. P. (20io) The effect of deliberate play on tactical performance in basketball. Perceptual \& Motor Skills, IIо, 849-856. http://dx.doi.org/Io.2466/pms.IIO.3.849-856

Gulbin, J. P. (2006) Why deliberate practice isn't enough? Paper presented to the Symposium on "Dimensions of Performance». Berlin, Alemania. Recuperado el 8 de agosto de 2orI, de www.icsspe.org/download/documente/sonstiges/Gulbin.doc. 
Gulbin, J. P. (2008) Identifying and developing sporting experts. En D. FArRow, J. BaKer y C. MacMahon (eds.) Developing sport expertise (pp. 6o-72). Abingdon: Routledge.

Gulbin, J. P.; Croser, M. J.; Morley, E. J. y Weissensteiner, J. R. (2013) An integrated framework for the optimization of sport and athlete development: A practitioner approach. Journal of Sports Sciences. http://dx.doi.org/I0.1080/026404I4.2013.78166I

Gulbin, J. P.; Oldenziel, K. E.; Weissensteiner, J. R. y Gagné, F. (20io) A look through the rear view mirror: Developmental experiences and insights of high performance athletes. Talent Development \& Excellence, 2 (2), I49-I64.

Gulbin, J. P.; Weissensteiner, J. R.; Oldenziel, K. E. y Gagné, F. J. (2013) Patterns of performance development in elite athletes. European Journal of Sport Science. http://dx.doi.org/I0.1080/17461391.20I2.756542

Güllich, A. (2007) Training - Support - Success: Control-related assumptions and empirical findings. Saarbruücken: University of the Saarland.

GÜLLICH, A. (2013) Selection, de-selection and progression in German football talent promotion. European Journal of Sport Science. http://dx.doi.org/10.1080/17461391.2013.858371

Güllich, A. (20I4) Many roads lead to Rome - Developmental paths to Olympic gold in men's field hockey. European Journal of Sport Science, I4 (8), 763-771. http://dx.doi.org/10.1080/17461391.2014.905983

Güllich, A. y EMrich, E. (2006a) Evaluation of the support of young athletes in the elite sport system. European Journal of Sport and Society, 3 (2), 85-108.

Güllich, A. y Emrich, E. (2006b) Sport-spanning training variability augments individual sucess potencial in elite sport. En H. Hoppeler, T. Reilly, E. Tsolakidis, L. Gfeller y S. Klossner (eds.) Book of abstracts of the inth Annual Congress of the European College of Sport Science (p. 533). Cologne: Sportverlag Strauss.

Güllich, A. y Emrich, E. (20I2) Considering long-term sustainability in the development of world class success. European Journal of Sport Science. http://dx.doi.org/IO.I080/17461391.2012.706320

Hambrick, D. Z.; Oswald, F. L.; Altmann, E. M.; Meinz, E. J.; Gobet, F. y Campitelli, G. (2013) Deliberate practice: Is that all it takes to become and expert? Intelligence, 45, 34-45. http://dx.doi.org/IO.IoI6/j.intell.2013.04.00I

Helsen, W. F.; Starkes, J. L. y Hodges, N. J. (1998) Team sports and the Theory of Deliberate Practice. Journal of Sport and Exercise psychology, 20, 12-34.

Hoare, D. G. y Warr, C. R. (2000) Talent identification and women's soccer: An Australian experience. Journal of Sports Sciences, I8, 751-758. http://dx.doi.org/Io.I080/02640410050I20122

Hodge, T. y Deakin, J. (1998) Deliberate practice and expertise in the martial arts: The role of context in motor recall. The Journal of Sport and Exercise Psychology, 20, 260-279.

Hodges, N. J. y STARKes, J. L. (I996) Wrestling with the nature of expertise; a sport specific test of Ericsson, Krampe and Tesch-Roemer's (1993) theory of deliberate practice. International Journal of Sport Psychology, 27 (4), 400-424.

Howe, M. J. A.; Davidson, J. W. y Sloboda, J. A. (1998) Innate talents: Reality or Myth? Behavioral and Brain Sciences, 21, 399-407. http://dx.doi.org/ı0.1017/Sor40525X9800123X

Karalejic, M.; Jakovljevic, S. y Macura, M. (20II) Anthropometric characteristics and technical skills of $\mathrm{I} 2$ and $\mathrm{I} 4$ year old basketball players. Journal of Sports Medicine and Physical Fitness, 51, IO3-IIO.

Leite, N.; Baker, J. y Sampaio, J. (2009) Paths to expertise in Portuguese national team athletes. Journal of Sport Science and Medicine, 9 (8), 560-566.

Li, Ch.; John Wang, C. K. y Young Pyun, D. (20I4) Talent development environmental factors in sport: A review and taxonomic classification. Quest, 66, 433-447. http://dx.doi.org/10.1080/00336297.2014.944715 
Lidor, R.; Côté, J. y Hackfort, T. (2009) IssP Position Stand: To test or no to test? The use of physicall skill tests in talent detection and in early phase of sport development. International Journal of Sport and Exercise Psychology, 7, 3I-I46.

Lidor, R.; Falk, B.; Arnon, M.; Cohen, Y.; Segal, G. y Lander, Y. (2005a) Measurement of talent in team-handball: The questionable use of motor and physical tests. Journal of Strength and Conditioning Research, 19, 318-325.

http://dx.doi.org/IO.I519/1533-4287(2005)19[318:MOTITH]2.o.CO;2 http://dx.doi.org/I0.1519/00I24278-200505000-000I4

Lidor, R.; Hershko, Y.; Bilkevitz, A.; Arnon, M. y Falk, B. (2007) Measurement of talent in volleyball: 15 -month follow-up of elite adolescent players. The Journal of Sports Medicine and Physical Fitness, 47, 159-168.

Lidor, R.; Melnik, Y.; Bilkevitz, A.; Arnon, M. y Falk, B. (2005b) Measurement of talent in judo using a unique, judo-specific test. The Journal of Sports Medicine and Physical Fitness, $45,32-37$.

Lorenzo, A. (2003) ¿Detección o Desarrollo del Talento? Factores que motivan una nueva orientación del proceso de detección de talentos. Apunts, Educación Física y Deporte, 71, 23-28.

Lorenzo, A.; Borrás, P.; SÁnchez, J. M.; Jiménez, S. y SAmpedro, J. (2009) Career transition from junior to senior in basketball players. Revista de Psicología del Deporte, I8, 309-312.

Lorenzo, A. y Calleja, J. (2010) Factores condicionantes del desarrollo deportivo. Bilbao: Diputación Foral de Vizcaya.

LorenZo, A.; JimÉnEZ, S. y Lorenzo, J. (20I4) ¿Son realmente eficaces los programas de detección de talentos deportivos? Nuevos horizontes para su diseño. Kronos, I3 (I).

Lorenzo, A. y SAmpaio, J. (2005) Reflexiones sobre los factores que pueden condicionar el desarrollo de los deportistas de alto nivel. Apunts Educación Física y Deportes, 80 (2), 63-70.

Macnamara, B. N.; Hambrick, D. Z. y Oswald, F. L. (20I4) Deliberate Practice and Performances in Music, Games, Sports, Education, and Professions: A Meta-Analysis. Psychological Science.

http://dx.doi.org/IO.II77/0956797614535810

Matthys, S. P. J.; Vaeyens, R.; Vandendriessche, J.; Vandorpe, B.; Pion, J.; CoutTs, A. J.; Lenoir, M. y Philippaerts, R. M. (20Ii) A multidisciplinary identification model for youth handball. European Journal for Sport Science, II (5), 355-363. http://dx.doi.org/ı0.1080/17461391.2010.523850

Memmert, D.; Baker, J. y Bertsch, C. (20Io) Play and practice in the development of sportspecific creativityin team ball sports. High Ability Studies, 2I (I), 3-18. http://dx.doi.org/10.1080/13598139.2010.488083

Meylan, C.; Cronin, J.; Oliver, J. y Hughes, H. (20Io) Talent identification in soccer: The role of maturity status on physical, physiological and technical characteristics. International Journal of Sport Science and Coaching, 5 (4), 571-592.

http://dx.doi.org/IO.I260/1747-954I.5.4.57I

Moesch, K.; Trier Hauge, M. L.; Wikman, J. M. y Elbe, A. M. (2013) Making it to the top in team sports: Start later, intensify, and be determined! Talent Development E Excellence, 5 (2), 85-100.

MORRIS T. (2000) Psychological characteristics and talent identification in soccer. Journal of Sports Sciences, I8 (9), 715-726. http://dx.doi.org/I0.I080/02640410050I20096

Mosing, M. A.; Madison, G.; Pedersen, N.; Kuja-Halkola, R. y Ullén, F. (20I4) Practice does not make perfect no causal effect of music practice on music ability. Psychological Science. http://dx.doi.org/ı.1177/0956797614541990

Oldenziel, K.; Gagné, F. y Gulbin, J. P. (2004) Factors affectin the rate of athlete development from novice to senior elite: how applicable is the io-year-rule? Comunicación presentada en el 2004 Pre-Olympic Congress - «Sport Science Through the ages». Thessaloniki, 
Grecia. Recuperado el 20 de septiembre de $201 \mathrm{~d}$ de http://cev.org.br/biblioteca/factorsaffecting-the-rate-of-athlete-development-from-novice-to-senior-elite-how-applicable-isthe-Io-year-rule/.

Pearson, D. T.; Naugthon, G. A. y Torode, M. (2006) Predictability of physiological testing and the role of maturation in talent identification for adolescent team sports. Journal of Science and Medicine in Sport, 9, 277-287. http://dx.doi.org/IO.I0I6/j.jsams.2006.05.020

Phillips, E.; Keith, D.; Renshaw, I. y Portus, M. (20I0) Expert performance in sport and the dynamics of talent development. Sports Medicine, 40 (4), 271-283. http://dx.doi.org/I0.2165/I1319430-000000000-00000

PienaAR, A. E. y SPAMER, E. J. (I998) A longitudinal study of talented young rugby players as regards their rugby skills, physical and motor abilities and anthropometric data. Journal of Human Movement Studies, 34 (I), I3-32.

Reilly, T.; Bangsbo, J. y Franks, A. (2000a) Anthropometric and physiological predispositions for elite soccer. Journal of Sports Sciences, 8, 669-683. http://dx.doi.org/I0.1080/02640410050I20050

Reilly, T.; Williams, A. M.; Nevill, A. y Franks, A. (20oob) A multidisciplinary approach to talent identification in soccer. Journal of Sports Sciences, 8, 695-702. http://dx.doi.org/I0.1080/02640410050120078

Ruiz, L. M.; SÁnchez, M.; Durán, J. P. y Jiménez, C. (2006) Los expertos en el deporte: Su estudio y análisis desde una perspectiva psicológica. Anales de Psicología, I (22), I32-I42.

SÁenz-López, P.; Ibáñez, S. J.; Giménez, J.; Sierra, A. y SÁnchez, M. (2005) Multifactor characteristics in the process of development of the male expert basketball player in Spain. International Journal of Sport Psychology, 36, 151-171.

Schumacher, Y. O.; Mroz, R.; Mueller, P.; Schmid, A. y Ruecker, G. (2006) Succes in elite cycling: A prospective and retrospective analysis of race results. Journal of Sports Sciences, 24 (II), II49-II56.

Simonton, D. K. (1999) Talent and its development: An emergenic and epigenetic model. Psychological Review, 106, 435-457. http://dx.doi.org/Io.1037/0033-295X.106.3.435

Simonton, D. K. (200I) Talent development as a multidimensional, multiplicative, and dynamic process. Current Directions in Psychological Science, Io, 39-43. http://dx.doi.org/ı0.IIII/I467-8721.0oIIo

Singer, R. N. y Janelle, C. H. (I999) Determining sport expertise: From genes to supremes. International Journal of Sport Psychology, 2 (30), II7-I50.

SOBERLAK, P. y CÔTÉ, J. (2003) The developmental activities of elite ice hockey players. Journal of Applied Sport Psychology, 15, 4I-49.

http://dx.doi.org/I0.IO80/I04I320030540I

Stambulova, N. y Alfermann, D. (2009) Putting culture into context: Cultural and cross-cultural perspectives in career development and transition research and practice. International Journal of Sport and Exercise Psychology, 292-308. http://dx.doi.org/I0.1080/ı612197X.2009.967191I

STARKES, J. L. (2000) The road to expertise: Is practice the only determinant? International Journal of Sport Psychology, 3I, 43I-45I.

Starkes, J.; Deakin, J.; Allard, F.; Hodges, N. y Hayes, A. (1996) Deliberate practice in sports: What is it anyway? En K. A. ERICSSON (ed.) The road to excellence. The acquisition of expert performance in the arts and sciences, sport and games (pp. 8I-Io6). New Jersey: LEA.

STRACHAN, L.; Côté, J. y DeAKIN, J. (2009) «Specializers» versus «Samplers»in youth sport: comparing experiences and outcomes. The Sport Psychologist, 23, 77-92.

Stroyer, J.; Hansen, L. y Hansen, K. (2004) Physiological profile and activity pattern of young soccer players during match play. Medicine and Science Sports Exercise, 36 (I), I68-174. http://dx.doi.org/I0.1249/oI.MSS.ooooro6187.05259.96 
Torres-Unda, J.; Zarrazquin, I.; Gil, J.; Ruiz, F.; Irazusta, A.; Kortajarena, M.: Seco, J. y IRAZUSTA, J. (2013) Anthropometric, physiological and maturational characteristics in selected elite and non-elite male adolescent basketball players. Journal of Sports Sciences, 3I (2), 196-203. http://dx.doi.org/IO.I080/026404I4.2012.725133

Tranckle, T. y Cushion, C. J. (2006) Rethinking giftedness and talent in sport. Quest, 8, 65-282.

http://dx.doi.org/Io.1080/00336297.2006.10491883

Tucker, R. y Collins, M. (20I2) What makes champions? A review of the relative contribution of genes and training to sporting success. British Journal of Sports Medicine, 46, 555-56I. http://dx.doi.org/Io.II36/bjsports-20II-090548

Vaeyens, R.; Güllich, A.; Warr, Ch. R. y Philippaerts, R. (2009) Talent identification and promotion programmes of Olympic athletes. Journal of Sports Sciences, 27 (13), 1367-1380. http://dx.doi.org/I0.1080/026404I0903110974

Vaeyens, R.; Lenoir, M.; Williams, A. M. y Phillippaerts, R. M. (2008) Talent identification and development programmes in sport: current models and future directions. Sports Medicine, 38 (9), 703-7I4. http://dx.doi.org/I0.2165/00007256-200838090-0000I

Vaeyens, R.; Malina, R. M.; Janssens, M.; Van Renterghem, B.; Bourgois, J.; Vrijens, J. y Philippaerts, R. M. (2006) A multidisciplinary selection model for youth soccer: the Ghent Youth Soccer Project. British Journal of Sports Medicine, 40 (II), 928-934. http://dx.doi.org/ı.1136/bjsm.2006.029652

Vandorpe, B.; Vandendriessche, J. B.; Vaeyens, R.; Pion, J.; Lefebre, J.: Philippaerts, R. M. y LenOIR, M. (20I2) The value of a non-sport-specific motor test battery in predicting performance in young female gymnasts. Journal of Sports Sciences, 30 (5), 497-505. http://dx.doi.org/I0.1080/026404I4.2012.654399

Van Rossum, J. H. y Gagné, F. (2005) Talent development in sports. En F. A. Dixon y S. M. Moon (eds.) The Handbook of Secondary Gifted Children. (pp. 28I-316). Waco: Prufrock Press.

Ward, P.; Hodges, N. J.; Starkes, J. L. y Williams, A. M. (2007) The road to excellence in soccer: A quasi-longitudinal approach to deliberate practice. High Ability Studies, I8, I19-153. http://dx.doi.org/I0.1080/13598130701709715

Williams, A. M. y Franks, A. (1998) Talent identification in soccer. Sports Exercise and Injury, 4, I59-I65.

Williams, A. M. y Ford, P. R. (2008) Expertise and expert performance in sport. International Review of Sport and Exercise Psychology, I, 4-18. http://dx.doi.org/ıo.1080/I7509840701836867

Williams, A. J. y Hodges, N. J. (2005) Practice, instruction and skill acquisition in soccer: Challenging tradition. Journal of Sports Sciences, 23 (6), 637-650. http://dx.doi.org/I0.1080/02640410400021328

Williams, A. M. y Reilly, T. (2000) Talent identification and development in soccer. Journal of Sports Sciences, 8, 657-667. http://dx.doi.org/I0.1080/02640410050I2004I

Zibung, M. y Conzelmann, A. (20I4) The role of specialization in the promotion of young football talents: A person-oriented stuyde. European Journal of Sport Science, I3 (5), 452-460. http://dx.doi.org/IO.IO80/17461391.2012.749947 